\title{
Corrigendum
}

Genes Genet. Syst. (2014) 89, p. 227-235

\section{Mitochondrial genetic diversity, phylogeny and population structure of Hydropotes inermis in South Korea}

Baek-Jun Kim, Yun-Sun Lee, Yong-Su Park, Kyung Seok Kim, Mi-Sook Min, Sang-Don Lee and Hang Lee

The first sentence in the acknowledgment on p. 234 should be changed to "This study was supported in part by the Research Institute for Veterinary Science, BK21 PLUS Program for Creative Veterinary Science Research, Seoul National University and the Korea Science and Engineering Foundation (KOSEF) grant funded by the Korean government (MEST) (No. 2009-0080227)." 